\title{
Real-time Augmented Reality shopping platform for studying consumer cognitive experiences
}

\author{
Jasmina Stoyanova, Ricardo Gonçalves, António Coelho \\ Faculdade de Engenharia da Universidade do Porto FEUP \\ Portugal 4200-465 Porto Rua Dr. Roberto Frias 378 \\ Email: pdd10007@fe.up.pt,refg@fe.up.pt, acoelho@fe.up.pt
}

\author{
Pedro Brito \\ Faculdade de Economia FEP \\ Portugal 4200-464 Porto Rua Dr. Roberto Frias \\ Email: pbrito@fep.up.pt
}

\begin{abstract}
Augmented Reality (AR) is a technology which produces a synthesis between a computer-generated data and the physical world of a viewer while establishing 3D registration and real time interaction. Among the wide range of application of $\mathrm{AR}$, its use in advertising shopping experiences has recently been embraced by advertisers due to its novelty and engaging potential. Part of a wider research aiming at understanding the impact of AR on consumer psychology, this paper presents a demo platform application developed for a real-time shopping experience for shoes and attempts to define a ground base for posterior marketing research in the field. In order to fully evaluate consumer experiences and compare with the main AR platform two other shopping applications were designed: a marker-based and a static one. The platform will assist in exploring the antecedents of consumer purchase intention and in defining metrics for measuring shopping experiences with AR.
\end{abstract}

\section{INTRODUCTION}

Advertising is a form of communication that aims to inform, persuade or encourage a target audience into taking an action of purchase. In order to achieve its goal, advertising makes use of most of the human senses with the visual characteristics being especially important in a passive, one way transmission of a message. However, the messages delivered with traditional advertising such as print, broadcast and direct mail nowadays appear as much more restricting compared to newer forms of communications. Furthermore, conventional advertising does not allow consumers the liberty to interact freely with an ad or choose the flow of information they receive. Additionally, audiences are already fully well acquainted with traditional forms of publicity and to the point where no element of surprise or novelty exists. What ad strategy builders seek increasingly more and more are different forms of audience immersiveness and participation to create a deeper level of engagement in an original form. Since recently Augmented Reality has been gaining momentum in the advertising industry, an increasing number of companies have expressed interest in adopting it in their marketing approaches. An advantage of the technology is its ability to display interactively threedimensional representations of real objects (e.g. for product demonstrations) thus generating a more significant impact on the audience. The demo platform, described in this paper, aims to assist in studying the cognitive effects on consumers through an AR shopping application in comparison to two other applications: a marker-based and a static. The primary objective of this work is to obtain a thorough understanding of whether AR product presentation may engage the user at a greater degree and operate as a tool for enriching consumer ex- periences. The main contribution of the research is established in providing guidance for future practice in advertising and an attempt to interpret the impact of AR advertising on aspects of consumer psychology, such as emotions, arousal, consumer involvement, memory and knowledge. The study aims to assist in the formation of new consumer psychological profiles and to evaluate which characteristics best explain their cognitive and emotional reactions to AR. Since the project will be divided in two consecutive parts technological development and result gathering and evaluation - this paper will focus mainly on the tools and steps used for establishing a suitable platform for testing. Finally, due to the lack of scientific research in the field, the data collected will be instrumental in defining approaches in subsequent marketing studies, leading to a better understanding of consumer behavior and targeting of products and services.

\section{STATE OF THE ART}

At this moment Augmented Reality can be achieved by two main ways [3][4], either through a head mounted display (HMD) set up [1] or through combining a camera/sensor with a video screen as output [2]. The two commonly used types of HMDs are video and optical. Video HMDs are usually closed view devices with one or two mounted cameras which feed the users view. By intercepting the feed from these cameras it is then possible to add pertinent information associated with what is being seen. Optical HMDs on the other hand are devices which display information in a similar manner to the previous type except that the users can see through them. Google Glass is one of the most recent and lightweight examples of an optical HMD, currently under testing by Google X Lab, which aims to revolutionize and bring this type of technology to the masses. The other type of approach to AR involves either a camera or a sensor, or a combination of both. The data fed by these devices are processed in real time, combining it with other media, and the result image is outputted to a video screen such as a TV screen or a smart phone display. Using this approach, AR can appear in two forms (or combined): marker-based and markerless. Both forms of AR require at least a basic camera, however markerless AR may greatly benefit from using a motion sensor such as Kinect. Marker-based AR makes use of special printed symbols that are recognized by the application and are used as a spatial reference to place a model in the scene, usually on top of the marker, following and rotating accordingly. One of the downsides of this approach is that it requires an external factor, a marker, which may be sufficient for some applications 
and restricting for others. An example of a situation where it may be restrictive is public advertisement in a mall, which would require assistance in handing markers to customers or depending upon an installation of specific software on their smartphones. Furthermore, using a smartphone application approach would result in failure of reaching desired volume of publicity, leaving the customers who do not own a smart phone (or have not pre-installed the application) without an access to the advertisement. However, thanks to the available development kits, such as ARToolkit or Vuforia, it is fairly easy to implement good solutions. A recent advertisement example of marker based approach was District 9 AR Demo which showed different animated characters from the movie when a marker was visible to the camera1. On the other hand, the markerless approach, as the name indicates, does not make use of symbol markers, detecting instead features of its users, such as hands, head or even the full body skeleton. In spite of the development kits available, such as OpenCV, this form may prove to be quite more challenging in creating a good solution. In our first attempts at creating an AR application we tried OpenCV with a general web camera to track faces and hands using HAAR cascade files available on the development kit web site. In our tests face recognition was fairly good, however hands had to be in specific positions to be detected and both faces and hands would not be recognized if they slightly stopped facing the camera. Alternatively to using a common web camera, a motion sensor such as Microsoft Kinect can yield better results by tracking movement and mapping users skeletons into a virtual 3D representation. A clear use of this approach in marketing are the virtual cloth trying booths, such as Barbies Augmented Reality Dream Closet2, which allows users to try different clothes without actually putting them on.

\section{APPROACH}

After testing several approaches (both marker and markerless) to ascertain which would provide the desired conditions and that would enable us to perform the study with as little limitations as possible, we concluded that the Microsoft Kinect sensor would be the best option. The reasoning behind this choice can be broken down in two avenues. Firstly, the tracking system is quite robust and accurate, even if some of the tracked body elements get out of its visibility cone. Through this tracking system any user can interact with the application without using any external object, such as marker. Secondly, by coupling this device with the game engine Unity3D through the use of Kinect for Windows SDK, the implementation became fairly easy, allowing more time to focus on the actual experience the application is meant to provide. By using the SDK we were able to access the users skeleton map (hands, arms, head, etc.) and generate a 3D representation of all the skeleton elements through their position within the game engine. For this demo we focused only on tracking hands since it was sufficient for our purposes. By tracking the hands position we were then able to map their location to a $2 \mathrm{D}$ plane streaming the video from the Kinect camera. This is so that the users image in the $2 \mathrm{D}$ plane activates the right interface when they try to interact with any of the available interfaces.

\section{DEMO PROTOTYPE}

The demo application starts by showing a black screen with a humanoid image with the arms raised, instructing users

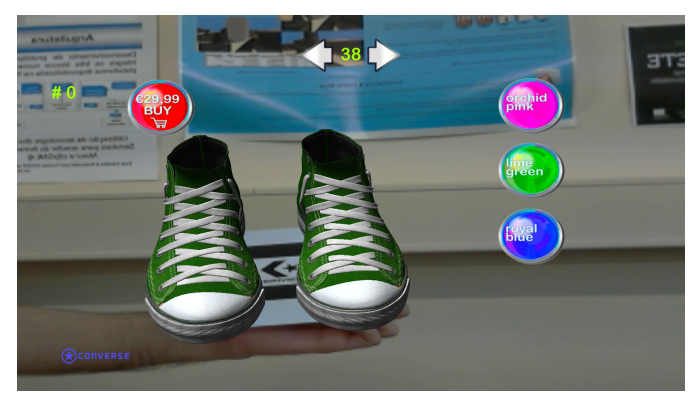

Fig. 1: Marker-based application

on how to activate it. This measure was taken in order to prevent users from seeing immediately what they were about to experience, and to record their expressions upon activation, as well as to let them know that they would have to interact with the application with their movements. Immediately after activation, a new screen is presented to the user composed by real time streaming of the Kinect captured video. For a product prototype a highly detailed 3D model of a pair of sneakers were chosen. Three interactive screen buttons allow changing the color and a button to make a purchase. The purchase buttons main objective is to store information on the amount of users who chose to purchase the sneakers and also to register the chosen color. The data are stored in a SQLite database which can be accessed later for statistical information. As mentioned above, there are three interfaces with which a user can interact: double hand activator, which requires both hands to trigger the application; four buttons which only require one hand to hover above the interface and a rotator, activated by moving a hand from down to up or from left to right while hovering the interface. For the execution of the first two interfaces the user must position the hand over for detection for two seconds while the buttons start to glow around increasingly until they are activated. To reactivate the same interface the hand must first be removed and placed over the selected interface once again. The other two applications are similar to the main one with the exception of how users interact with them. In the Marker based case(figure 1), markers are used instead of the Kinect sensor while the static application has no AR functionalities and users must interact with it in the traditional way (e.g. through a computer mouse).

\section{ACKNOWLEDGMENT}

The research leading to these results has received funding from the European Union Framework Programme (FP7/20072013) under grant agreement n 285417.

\section{REFERENCES}

[1] Azuma, Ronald T. "A survey of augmented reality." PresenceTeleoperators and Virtual Environments 6.4 (1997): 355-385.

[2] Prochazka, David, and Tomas Koubek. "Augmented Reality Implementation Methods in Mainstream Applications." arXiv preprint arXiv:1106.5569 (2011).

[3] Zlatanova, Sikya. "Augmented reality technology." GISt Report 17 (2002): 1-76.

[4] Van Krevelen, D. W. F., and R. Poelman. "A survey of augmented reality technologies, applications and limitations." International Journal of Virtual Reality 9.2 (2010): 1. 